C Revista de Matemática: Teoría y ApliCaCiones 2022 29(1) : 53-68

CIMPA - UCR ISSN: 1409-2433 (PRINT), 2215-3373 (ONLINE)

DOI: https://doi.org/10.15517/rmta.v29i1.40956

\title{
SOME APPLICATIONS OF PERIODIC ORBITS FOR COMPETITIVE SYSTEMS
}

\author{
ALGUNAS APLICACIONES DE ÓRBITAS \\ PERIÓDICAS A SISTEMAS COMPETITIVOS \\ HOMERo G. DíAZ-MARÍN * OSVALdo OSUNA ${ }^{\dagger}$
}

Received: 21/Oct/2020; Revised: 4/Apr/2021;

Accepted: 16/Sep/2021

Revista de Matemática: Teoría y Aplicaciones is licensed under a Creative Commons Attribution-NonCommercial-ShareAlike 4.0 International License.

http://creativecommons.org/licenses/by-nc-sa/4.0/

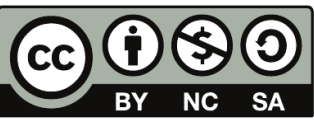

*Universidad Michoacana, Facultad de Ciencias Físico-Matemáticas, Morelia, México. E-Mail: hdiaz@umich.mx

${ }^{\dagger}$ Universidad Michoacana, Instituto de Física y Matemáticas, Morelia, México. E-Mail: osvaldo@ifm.umich.mx 


\begin{abstract}
We prove existence of periodic orbits for non-autonomous two dimensional competitive dynamical systems with periodic time dependence. The proof is an adaptation of a similar assertion stated for cooperative systems in [6]. We also give two main applications: one model for cancer cell populations under periodic chemotherapy as treated in [4] and [3] for the cooperative case, and another model for mosquito population replacement dynamics interacting with control sterile mosquitoes with periodic release [1], for the competitive case.
\end{abstract}

Keywords: competitive systems; periodic orbit; angiogenesis; cancer treatment modeling; population replacement modeling; Aedes aegypti; Wolbachia.

\title{
Resumen
}

Probamos la existencia de órbitas periódicas para sistemas dinámicos competitivos en dos dimensiones no autónomos con dependencia periódica respecto al tiempo. La prueba es una adaptación de un resultado similar para sistemas cooperativos en [6]. También damos dos aplicaciones: un modelo de población de celulas cancerosas sometidas a un tratamiento periódico de quimioterapia como se describe en [4] y [3] para el caso cooperativo, y otro modelo de poblaciones de mosquitos interactuando con mosquitos de control estériles liberados periódicamente [1], para el caso competitivo.

Palabras clave: sistemas competitivos; órbita periódica; angiogénesis; modelado de tratamientos de cáncer; modelo de reemplazo de población; Aedes aegypti; Wolbachia.

Mathematics Subject Classification: 34C25, 37C60, 37C10, 92D25, 92C50.

\section{Introduction}

Cooperative systems and competitive systems arise in the study of the interaction of two or more species. They share many qualitative properties such as the fact that the dynamics on limit sets can be reduced to $(n-1)$-dimensional cells, this was observed by Smale, see [5]. They have important differences as well. On one hand cooperative systems preserve the ordering of the initial conditions induced by the first quadrant cone, its flow also preserves this cone, see [5]. On the other hand, for competitive systems in three-dimensions, the flow does not preserve the ordering of initial conditions. This flow rather preserves the union of three different partial orderings, see [7]. 
Fortunately, for two-dimensional competitive systems there exists a suitable partial ordering preserved by the flow. We exploit this fact and adapt results due to Korman on the existence of periodic orbits arising between super- and sub-solutions, see [6].

One of the applications is a model, obtained by standard pharmacological kinematics arguments, that describes the dynamics of periodic doses in chemotherapy for cancer cell populations. In principle the two dimensional system (13) is neither competitive nor cooperative. Nevertheless, by a suitable change of variables, it becomes cooperative, see (14) below. In a previous work, see [2], we have described dynamics of cancer therapies such as radiotherapy, by using cooperative systems.

Another important application that illustrates our results is related to prevention models for the control Aedes aegypti mosquitoes, which are important vectors for several periodic diseases spread worldwide. Here we can describe the dynamics of mosquito populations competing with sterile mosquito populations released periodically. These control population is previously infected with Wolbachia bacteria. The competitive system with some conditions on the parameters yields a periodic orbit. We also describe some conditions for an orbit to converge to a periodic orbit.

\section{2D competitive systems}

Along this section we present the suitable adaptations of the results developed in [6] for the case of competitive systems.

Define the sud-est or (IV)-partial order as follows: For every $u=\left(u_{1}, u_{2}\right)$, $v=\left(v_{1}, v_{2}\right) \in \mathbb{R}^{2}$, we say that $u \leq_{\mathrm{IV}} v$, if $u_{1} \leq v_{1}$ and $u_{2} \geq v_{2}$. If $u \neq v$ we write $u<_{\text {IV }} v$. If $u_{1}<v_{1}$ and $u_{2}>v_{2}$, then we write $u \ll_{\text {IV }} v$. This partial order is explicitly defined in [7].

Consider a system

$$
\begin{aligned}
& \dot{x}=\mathrm{f}(t, x(t), y(t)), \\
& \dot{y}=\mathrm{g}(t, x(t), y(t)),
\end{aligned}
$$

where $\mathrm{f}, \mathrm{g}$ are $C^{1}$ in an open $D \subset \mathbb{R}^{2}$ and continuous $T$-periodic functions on $t$. Recall that (1) is said a competitive system in $\mathbb{R} \times D$ if

$$
\mathrm{f}_{y}(t, x, y) \leq 0, \text { and } \mathrm{g}_{x}(t, x, y) \leq 0, \forall t \in \mathbb{R},(x, y) \in D \text {. }
$$

for a brief introduction to competitive systems see [7, 5]. 
We say that a pair of $T$-periodic differentiable functions $(a(t), b(t))$ is a $(I V)$-sub-solution pair of (1) if

$$
\begin{aligned}
& \dot{a} \leq \mathrm{f}(t, a(t), b(t)), \\
& \dot{b} \geq \mathrm{g}(t, a(t), b(t)), \text { for all } t .
\end{aligned}
$$

Analogously a pair of $T$-periodic differentiable functions $(A(t), B(t))$ is a $(I V)$ super-solution pair if

$$
\begin{aligned}
& \dot{A} \geq \mathrm{f}(t, A(t), B(t)), \\
& \dot{B} \leq \mathrm{g}(t, A(t), B(t)), \text { for all } t .
\end{aligned}
$$

We say that sub- and super-solution pairs are ordered if for all $t$ we have $a(t)<$ $A(t)$ and $b(t)>B(t)$, i.e. if $(a(t), b(t)) \ll_{\mathrm{IV}}(A(t), B(t))$.

Lemma 2.1 Suppose that $(a(t), b(t)) \ll_{\mathrm{IV}}(A(t), B(t))$ are a sub- and supersolution pairs. Then the flow of competitive system (1) preserves the order $\ll_{\mathrm{IV}}$. That is, if a solution $(\mathrm{x}(t), \mathrm{y}(t))$ has initial condition such that

$$
\begin{array}{r}
a(0)<\mathrm{x}(0) \text { or } \mathrm{y}(0)<b(0), \text { and } \\
\mathrm{x}(0)<A(0), \text { or } B(0)<y(0),
\end{array}
$$

i.e. if $(a(0), b(0)) \ll_{\mathrm{IV}}(\mathrm{x}(0), \mathrm{y}(0)) \ll_{\mathrm{IV}}(A(0), B(0))$. Then

$$
(a(t), b(t)) \ll_{\mathrm{IV}}(\mathrm{x}(t), \mathrm{y}(t)) \ll_{\mathrm{IV}}(A(t), B(t)), \quad \forall t \geq 0,
$$

in other words,

$$
\begin{aligned}
& a(t)<\mathrm{x}(t)<A(t), \\
& B(t)<\mathrm{y}(t)<b(t) .
\end{aligned}
$$

This claim appears for instance in [5], although it only mentions that competitive systems for unrelated initial conditions solutions remain unrelated in time. Here unrelated refers to the partial order of cooperative systems. We adapt the proof of [6].

Proof of Lemma 2.1. To see this define $w(t):=\mathrm{x}(t)-a(t)$, so that

$$
\begin{aligned}
\dot{w} & =\mathrm{f}(t, \mathrm{x}, \mathrm{y})-\dot{a} \\
& =\mathrm{f}(t, a, b)-\dot{a}+\partial_{x} \mathrm{f}\left(t, \mathrm{x}^{\theta_{0}}, \mathrm{y}^{\theta_{0}}\right)(\mathrm{x}-a)+\partial_{y} \mathrm{f}\left(t, \mathrm{x}^{\theta_{0}}, \mathrm{y}^{\theta_{0}}\right)(\mathrm{y}-b) \\
& \geq \partial_{x} \mathrm{f}\left(t, \mathrm{x}^{\theta_{0}}, \mathrm{y}^{\theta_{0}}\right)(\mathrm{x}-a)+\partial_{y} \mathrm{f}\left(t, \mathrm{x}^{\theta_{0}}, \mathrm{y}^{\theta_{0}}\right)(\mathrm{y}-b)=p w+q z
\end{aligned}
$$


with $\mathrm{x}^{\theta}(t)=\theta \mathrm{x}(t)+(1-\theta) a(t), \mathrm{y}^{\theta}(t)=\theta \mathrm{y}(t)+(1-\theta) b(t), \forall \theta \in[0,1]$, and where $z(t):=\mathrm{y}(t)-b(t)$ and $q(t) \leq 0$. Analogously,

$$
\begin{array}{cc}
\dot{w} \geq p w+q z, & w(0)>0, \\
\dot{z} \leq r w+s z, & z(0)<0,
\end{array}
$$

where $r(t) \leq 0$. Hence, for $\mu(t)=e^{-\int^{t} p}>0, \nu(t)=e^{-\int^{t} s}>0$, we have

$$
\frac{d}{d t}(\mu w) \geq \mu q z, \quad \frac{d}{d t}(\nu z) \leq \nu r w .
$$

We claim that $w$ is monotone increasing with $w(0)>0$ and that $z$ is monotone decreasing with $z(0)<0$. We proceed by contradiction.

Suppose that $t_{0}:=\inf \{t>0: w(t)<0\} \geq 0$. Then there exists an interval $\left(t_{0}, t_{0}+\epsilon\right)$, where $w(t), \dot{w}(t)<0$. Hence

$$
\dot{\mu} w>\mu \dot{w}+\dot{\mu} w \geq \mu q z, \quad \forall t_{0}<t<t_{0}+\epsilon .
$$

Therefore $0>q z+p w$. Suppose that $z(t) \leq 0$. Since $q \leq 0$, then $0>p w$. Hence $p>0$. Thus $0>q z$. This yields a contradiction $z(t)>0$.

On the other hand, suppose that $z(t)>0$ for every $t \in\left(t_{0}, t_{0}+\epsilon\right)$. Then, for $t_{1}:=\inf \{t>0: z(t)>0\} \geq 0$, we would have $t_{1} \leq t_{0}$. Similarly we can prove that $t_{1} \geq t_{0}$, hence $t_{0}=t_{1}$.

Notice that by continuity $w\left(t_{0}\right)=z\left(t_{0}\right)=0$. By (7) we also have $\dot{w}\left(t_{0}\right)=$ $\dot{z}\left(t_{0}\right)=0$. This would yield

$$
\dot{\mathrm{x}}\left(t_{0}\right)=\dot{a}\left(t_{0}\right), \dot{\mathrm{y}}\left(t_{0}\right)=\dot{b}\left(t_{0}\right), \mathrm{x}\left(t_{0}\right)=a\left(t_{0}\right), \mathrm{y}\left(t_{0}\right)=b\left(t_{0}\right) .
$$

Nevertheless, for $0 \leq t \leq t_{0}$ we have $w(t) \geq 0$ and $z(t) \leq 0$. Thus, by (7) $\dot{w}(t) \geq 0$, and $\dot{z}\left(t_{0}\right) \geq 0$. By monotonicity, and the initial conditions, $w(0)>0$ and $z(0)<0$, we reach a contradiction, namely that $\mathrm{x}\left(t_{0}\right)>a\left(t_{0}\right)$ and $\mathrm{y}\left(t_{0}\right)<b\left(t_{0}\right)$.

We have proved that $w, z$ are monotone increasing and decreasing respectively with $w(0)>0, z(0)<0$. Therefore $w(t)>0$ and $z(t)<0$, for all $t \geq 0$. This proves a couple of inequalities in (6). The other couple can be proved analogously.

The following Theorem is a direct extension of the result for cooperative systems proved in [6] for the case of competitive systems. 
Theorem 2.2 Assume that the system (1) is competitive and has (IV)-ordered sub-and super-solution pairs $(a(t), b(t)) \ll_{\mathrm{IV}}(A(t), B(t))$. Then the system has a T-periodic solution $(x(t), y(t))$, satisfying

$$
a(t) \leq x(t) \leq A(t), b(t) \geq y(t) \geq B(t), \quad \forall t \geq 0,
$$

whenever there is no fixed point $\left(x_{0}, y_{0}\right)$,

$$
(a(0), b(0)) \leq_{\mathrm{IV}}\left(x_{0}, y_{0}\right) \equiv(x(t), y(t)) \leq_{\mathrm{IV}}(A(0), B(0))
$$

such that for every sub-super-solution pair we have

$$
a(t) \leq x_{0} \leq A(t), \quad B(t) \leq y_{0} \leq b(t) .
$$

Furthermore, any solution of (1), with initial condition $(\mathrm{x}(0), \mathrm{y}(0))$ satisfying $a(0)<\mathrm{x}(0)<A(0)$ and $b(0)>\mathrm{y}(0)>B(0)$, converges to the product of the strips

$$
(\check{x}(t), \hat{x}(t)) \times(\hat{y}(t), \check{y}(t)),
$$

where $(\check{x}(t), \check{y}(t)) \ll_{\mathrm{IV}}(\hat{x}(t), \hat{y}(t))$ are (IV)-minimal, maximal T-periodic solution, respectively.

Notice that we have added an implicit condition that does not appear explicitly in [6] about non-existence of certain points, in which case we could have an attracting fixed point for every initial condition, $a(0)<\mathrm{x}(0)<A(0), B(0)<$ $\mathrm{y}(0)<b(0)$. See an example of this phenomenon in [2].

Furthermore, another imprecise statement in [6] is that there are strict inequalities in the corresponding inequalities for the cooperative case as in (8). Nevertheless, there can be some equalities as it actually happens in Theorem 4.2 and in the examples of Section 4 below.

As conclusion for the previous critics and discussion, we suggest that the original statement in [6], should incorporate explicitly an additional hypothesis, namely that there are no $\left(x_{0}, y_{0}\right)$ such that one or two of inequalities in (9) adapted for the cooperative case, for every super-solution sub-solution pair.

Our result stated in Theorem 2.2 generalizes the result given in [6] for competitive systems instead of cooperative systems, that is we use the partial ordering, $\leq_{\mathrm{IV}}$, instead of the usual partial ordering of cooperative systems described therein.

Proof of Theorem 2.2. We first prove the existence of periodic solutions. Take $x_{0}=a, y_{0}=b$. Let $x_{1}(t), y_{1}(t)$ be the $T$-periodic solutions of

$$
\begin{aligned}
& \dot{x}_{1}+M x_{1}=\mathrm{f}_{1}\left(t, x_{0}, y_{0}\right)=M x_{0}+\mathrm{f}\left(t, x_{0}, y_{0}\right), \\
& \dot{y}_{1}+M y_{1}=\mathrm{g}_{1}\left(t, x_{0}, y_{0}\right)=M y_{0}+\mathrm{g}\left(t, x_{0}, y_{0}\right),
\end{aligned}
$$


where $M>0$ will be chosen later. On one hand we have that $M\left(x_{1}-a\right)=$ $\mathrm{f}(t, a, b)-\dot{x}_{1} \geq \dot{a}-\dot{x}_{1}$ or $\frac{d}{d t}\left(x_{1}-a\right)+M\left(x_{1}-a\right)=: \alpha_{1}(t) \geq 0$. Hence, $x_{1}-a \geq 0$, more explicitly,

$$
x_{1}(0)-a(0)=\frac{1}{e^{M T}-1} \int_{0}^{T} e^{M s} \alpha_{1}(s) d s \geq 0 .
$$

On the other hand for $y_{1}$ we have $M\left(y_{1}-b\right)=\mathrm{g}\left(t, x_{0}, y_{0}, z_{0}\right)-\dot{y}_{1} \leq \dot{b}-\dot{y}_{1}$ or $\frac{d}{d t}\left(y_{1}-b\right)+M\left(y_{1}-b\right)=: \beta_{1}(t) \leq 0$. Hence $y_{1} \leq b$. By induction we can consider the $T$-periodic solutions for $n \geq 1$ :

$$
\begin{aligned}
& \dot{x}_{n+1}+M x_{n+1}=\mathrm{f}_{n+1}\left(t, x_{n}, y_{n}, z_{n}\right)=M x_{n}+\mathrm{f}\left(t, x_{n}, y_{n}\right), \\
& \dot{y}_{n+1}+M y_{n+1}=\mathrm{g}_{n+1}\left(t, x_{n}, y_{n}, z_{n}\right)=M y_{n}+\mathrm{g}\left(t, x_{n}, y_{n}\right),
\end{aligned}
$$

where

$$
\begin{array}{r}
\frac{d}{d t}\left(x_{n+1}-x_{n}\right)+M\left(x_{n+1}-x_{n}\right)=\alpha_{n} \\
\alpha_{n}=M\left(x_{n}-x_{n-1}\right)+\mathrm{f}\left(t, x_{n}, y_{n}\right)-\mathrm{f}\left(t, x_{n-1}, y_{n-1}\right), \\
\frac{d}{d t}\left(y_{n+1}-y_{n}\right)+M\left(y_{n+1}-y_{n}\right)=\beta_{n} \\
\beta_{n}=M\left(y_{n}-y_{n-1}\right)+\mathrm{g}\left(t, x_{n}, y_{n}\right)-\mathrm{g}\left(t, x_{n-1}, y_{n-1}\right) .
\end{array}
$$

Take $M+\inf _{C}\left\{\partial_{x} \mathrm{f}(t, x, y)\right\}>0$ and $M+\inf _{C}\left\{\partial_{y} \mathrm{~g}(t, x, y)\right\}>0$ for

$$
C=\{(t, x, y): x \in[a(t), A(t)], y \in[B(t), b(t)], t \in[0, T]\} .
$$

With this choice and because of $(1)$ we have that $\mathrm{f}_{n}(t, x, y)$ is strictly increasing in $x$ and monotone decreasing on $y$; meanwhile $\mathrm{g}_{n}(t, x, y)$ is strictly increasing in $y$ and monotone decreasing on $x$. Thus

$$
\frac{d}{d t}\left(x_{n+1}-x_{n}\right)+M\left(x_{n+1}-x_{n}\right) \geq 0
$$

and

$$
\frac{d}{d t}\left(y_{n+1}-y_{n}\right)+M\left(y_{n+1}-y_{n}\right) \leq 0
$$

Therefore $x_{n+1} \geq x_{n}$, and $y_{n+1} \leq y_{n}$. In a similar way we obtain a sequence, $(a, b) \leq_{\mathrm{IV}}\left(x_{1}, y_{1}\right) \leq_{\mathrm{IV}} \ldots\left(x_{n}, y_{n}\right) \leq_{\mathrm{IV}}\left(X_{n}, Y_{n}\right) \leq_{\mathrm{IV}} \ldots\left(X_{1}, Y_{1}\right) \leq_{\mathrm{IV}}(A, B)$

If we take the sequence of $T$-periodic functions

$\underline{x}(t)=\lim _{n \rightarrow \infty} x_{n}(t), \underline{y}(t)=\lim _{n \rightarrow \infty} y_{n}(t), \bar{x}(t)=\lim _{n \rightarrow \infty} X_{n}(t), \bar{y}(t)=\lim _{n \rightarrow \infty} Y_{n}(t)$. 
Then $(\underline{x}(t), \underline{y}(t)),(\bar{x}(t), \bar{y}(t))$ are $T$-periodic. Moreover,

$$
(a(t), b(t)) \leq_{\mathrm{IV}}(\underline{x}(t), \underline{y}(t)) \leq_{\mathrm{IV}}(\bar{x}(t), \bar{y}(t)) \leq_{\mathrm{IV}}(A(t), B(t))
$$

By Cauchy convergence they are solutions of (1). This proves the existence of periodic solutions, whenever they do not correspond to fixed points.

The non-empty set of $T$-periodic orbits such that

$$
(a(0), b(0)) \ll_{\mathrm{IV}}(x(0), y(0)) \ll_{\mathrm{IV}}(A(0), B(0))
$$

is totally ordered by $\leq_{\mathrm{IV}}$. Therefore we can consider, $(\check{x}(t), \check{y}(t)),(\hat{x}(t), \hat{y}(t))$, as the minimal and maximal $T$-periodic solutions respectively.

We now prove that all solutions $(\mathrm{x}(t), \mathrm{y}(t))$, such that 5 holds, are attracted to a periodic solution. In fact we first prove that for every $n \in \mathbb{N}$ there exists $t_{n}>0$ such that

$$
\left(x_{n}(t), y_{n}(t)\right) \ll_{\mathrm{IV}}(\mathrm{x}(t), \mathrm{y}(t)) \ll_{\mathrm{IV}}\left(X_{n}(t), Y_{n}(t)\right), \quad \forall t \geq t_{n} .
$$

We prove for instance that $\left(x_{n}(t), y_{n}(t)\right) \ll_{\mathrm{IV}}(\mathrm{x}(t), \mathrm{y}(t))$ for certain $t \geq t_{n}$. Take a non-periodic solution (x,y) of (1) and a non-periodic solution $(\xi, \eta)$ of (10) with initial conditions $(a(0), b(0))$. That is

$$
\begin{aligned}
\dot{\xi}+M \xi & =M a+\mathrm{f}(t, a, b), & & \xi(0)=a(0), \\
\dot{\eta}+M \xi & =M a+\mathrm{g}(t, a, b), & & \eta(0)=b(0),
\end{aligned}
$$

Then by Lemma 2.1, $\frac{d}{d t}(\mathrm{x}-\xi)+M(\mathrm{x}-\xi)=\alpha_{1}(t)>0$, while $\frac{d}{d t}(\mathrm{y}-\eta)+$ $M(\mathrm{y}-\eta)=\beta_{1}(t)<0$. Moreover, $\mathrm{x}(t)-\xi(t)>\mathrm{x}(0)-\xi(0)>0$ and $\mathrm{y}(t)-\eta(t)<\mathrm{y}(0)-\eta(0)<0$. In addition $\frac{d}{d t}\left(x_{1}-\xi\right)+M\left(x_{1}-\xi\right)=0$ therefore $\xi(t) \nearrow x_{1}(t)$ as $t \rightarrow \infty$. Similarly $\eta(t) \searrow y_{1}(t)$. Hence there exists $t_{1}>0$ such that $\forall t \geq t_{1}$,

$$
\begin{aligned}
& \mathrm{x}(t)-x_{1}(t)=\mathrm{x}(t)-\xi(t)+\xi(t)-x_{1}(t)>0, \\
& \mathrm{y}(t)-y_{1}(t)=\mathrm{y}(t)-\eta(t)+\eta(t)-y_{1}(t)<0 .
\end{aligned}
$$

By induction we can consider $t_{n}>t_{n-1}>\cdots>t_{1}>0$, such that $\mathrm{x}(t)>x_{n}(t)$ and $\mathrm{y}(t)<y_{n}(t)$ for $t \geq t_{n}$.

\section{Antiangiogenic periodic cancer therapy}

As an application of the results we have till now exposed we consider the development of tumor tissue under the hypothesis of growing vascular network inside 
the tumor mass. Under this consideration, the maximal capacity of the cell population $K$ becomes a dynamical variable. This process is called angiogenesis and has been modeled by Hahnfeldt et.al. in [4], see also references therein.

A modification of the original model appears in [3].

$$
\begin{aligned}
\dot{V} & =-\alpha V \ln \left(\frac{V}{K}\right), \quad V>0, K \geq 0, \\
\dot{K} & =b K-\lambda K-d K V^{2 / 3}-e K g(t)
\end{aligned}
$$

This modified system happens to be cooperative under a suitable change of variable. More precisely, with the change of variable $V=-W$ it becomes cooperative in the region of the quadrant II,

$$
D=\{(W, K): W \leq 0, K \geq 0\}
$$

we get

$$
\begin{aligned}
\dot{W} & =\alpha W \ln \left(\frac{-W}{K}\right), \quad W \leq 0, K \geq 0, \\
\dot{K} & =b K-\lambda K-d K W^{2 / 3}-e K g(t) .
\end{aligned}
$$

Theorem 3.1 (Existence) Assume $\alpha, \lambda, b, d, e>0$ and $g(t)$ is a non negative, non constant continuous $T$-periodic function. If

$$
b>\lambda+e g^{*} .
$$

Then there exists at least one T-periodic solution $(V(t), K(t))$ of 13 whose components are positive.

Proof. For a positively invariant region where the system is cooperative we consider

$$
R=\{(W, K) \in D: M \leq K,-m \leq W \leq 0)\}
$$

for constants $0<m<\mu:=\left(\frac{b-\lambda-e g^{*}}{d}\right)^{3 / 2}$ and $m<M$. For a super-solution take the fixed point solution,

$$
\begin{array}{lr}
\dot{A}=0, & A(0)=0, \\
\dot{B}=0, & B(0)=M,
\end{array}
$$

with $M>0$ to be chosen; replacing in (4) we can see that the first equation in (4) is clearly satisfied. For the second relation we need

$$
\dot{B}(t)=0 \geq M\left(-\lambda+b-d M^{2 / 3}-e g(t)\right)
$$


we choose $\left(\frac{b-\lambda-e g_{*}}{d}\right)^{3 / 2}<M$, then inequality (17) is satisfied. We immediately have that these functions satisfy inequalities (4). Therefore they constitute a super-solution in $R$.

For constructing a sub-solution $(a(t), b(t))$ in $D$, we take

$$
\begin{array}{rrr}
\dot{a}=0, & a(0)=-m, \\
\dot{b}=0, & b(0)=m .
\end{array}
$$

In order to satisfy both inequalities in (3), we choose $0<m<M$. Consequently, $(a(t), b(t))$ form a sub-solution.

Therefore the main result for cooperative systems in [6] can be applied. So there exists at least one $T$-periodic solution for system (13), which proves the result.

Till now we have proven the existence of a periodic orbit for (14) inside

$$
R=[-m, 0] \times[m, M], \quad 0<m<\mu .
$$

It is possible to prove the uniqueness of the periodic orbit inside it and extend this region for $M \rightarrow \infty$ and $m \rightarrow 0$.

Thus we recover the result related to for the original model [4], see also the numerical simulations therein.

\section{Mosquito population periodic replacement}

A bacterium Wolbachia living only in insects has been studied as an agent for control population of mosquito, such as Aedes aegypti, see [1]. Descendants of an infected male mosquito Aedes and a non-infected female die early in development. Thus infected males can be released in the environment as a tool against mosquitoes expansion, having less negative environmental affectations than insecticides.

We adapt the model [1] for a periodic liberation of infected males into the environment. Thus for a time dependent Wolbachia-free mosquito population

$n_{1}(t)$ and an infected population $n_{2}(t)$ introduced in the environment, we have a dynamical system:

$$
\begin{aligned}
& \dot{n}_{1}=b_{1} n_{1}\left(1-s_{h} \frac{n_{2}}{n_{1}+n_{2}}\right)\left(1-\frac{n_{1}+n_{2}}{K}\right)-d_{1} n_{1}, \\
& \dot{n}_{2}=b_{2} n_{2}\left(1-\frac{n_{1}+n_{2}}{K}\right)-d_{2} n_{2}+g_{2},
\end{aligned}
$$


where $g_{2}(t)$ is a bacteria $T$-periodic periodic release rate. The other constant parameters $\left(b_{i}, d_{i}\right), i=1,2$, are the birth and death rate of each subpopulation, while $s_{h} \in[0,1]$, encodes the cytoplasmatic incompatibility rate and $K>0$ is the maximal mosquito population capacity.

Lemma 4.1 If $0 \leq s_{h} \leq 1$, then the system (19) is competitive in the first quadrant, $n_{1} \geq 0, n_{2} \geq 0$. Moreover, the subregion $D$ with $n_{1} \leq\left(1-d_{1} / b_{1}\right) K, n_{2} \leq$ $K$ in the first quadrant is positively invariant and every initial condition in the first quadrant eventually reaches $D$.

As an application of our result for competitive systems we prove the existence of periodic evolution of two populations for certain conditions on the parameters. This is contained in the following assertion.

Theorem 4.2 Assume $b_{1}, b_{2}, d_{1}, d_{2}, K>0,0 \leq s_{h} \leq 1$, are constants and $g_{2}(t)$ is a non-negative continuous $T$-periodic function. If $b_{1} \geq d_{1}$ and $g_{2}^{*}=$ $\max _{[0, T]} g_{2}(t) \leq d_{2} K$, then there exists at least one $T$-periodic solution in $D$ of (19) with vanishing Wolbachia-free population $n_{1} \equiv 0$. Moreover, if

$$
g_{2 *} \geq\left(d_{2}-b_{2} / 4\right) K
$$

with $g_{2 *}=\min _{[0, T]} g_{2}(t)$, then every initial condition in the first quadrant converges towards this periodic orbit which is a global attractor.

Proof. For a super-solution pair take the fixed point solution,

$$
\begin{array}{ll}
\dot{A}=0, & A(0)=M, \\
\dot{B}=0, & B(0)=0,
\end{array}
$$

for some sufficiently small $0 \leq M \leq\left(1-\frac{d_{1}}{b_{1}}\right) K$. For constructing a subsolution $(a(t), b(t))$, we take

$$
\begin{array}{ll}
\dot{a}=0, & a(0)=0, \\
\dot{b}=-d_{2} b+\left(b_{2} K / 4+g_{2}\right), & b(0)=b_{0} .
\end{array}
$$

where

$$
\begin{aligned}
b_{0} & =\frac{1}{e^{d_{2} T}-1} \int_{0}^{T} e^{d_{2} s}\left(b_{2} K / 4+g_{2}(s)\right) d s \\
b & =b_{0} e^{-d_{2} t}+e^{-d_{2} t} \int_{0}^{t} e^{d_{2} s}\left(b_{2} K / 4+g_{2}(s)\right) d s .
\end{aligned}
$$


Therefore Theorem 2.2 applies, so there exists at least one $T$-periodic solution for system (19). Moreover, since $M \rightarrow 0$ yields a super-solution then in the limit, there exists a unique periodic orbit of the form

$$
\left(0, n_{2}(t)\right), t \in[0, T]
$$

To prove stability, notice that any initial condition

$$
0<n_{1}(0)<K, \quad 0<n_{2}(0)<b_{0}
$$

converges towards the periodic orbit. Thus if condition 20 holds, then $b_{0} \geq K$ and every orbit in $D$ and outside $D$ converges towards the a unique periodic orbit.

Condition 20, may be refined to the condition

$$
b_{0} \geq K
$$

and by the same arguments we can prove global stability. Notice that 20) yields a useful bound for the minimal resources employed in the replacement, which warranties elimination of Wolbachia-free mosquito population.

For instance we may give other useful criteria if we consider the mean amount of liberated infected population per period, $\bar{g}_{2}=\int_{0}^{T} g_{2}(s) d s / T$. In this case, $b_{0} \geq K$ is implied by the following condition

$$
\frac{\bar{g}_{2}}{e^{d_{2} T}-1}+\frac{T K b_{2}}{4} \geq d_{2} K
$$

On the other hand, if condition (20) is not true, then maybe $b_{0}<K$. Thus, for $n_{2}(0)<b_{0}$ the initial amount of Wolbachia-infected mosquitoes yield vanishing Wolbachia-free population, $n_{1} \rightarrow 0$. While for $n_{2}(0)>b_{0}$, there is no warranty that Wolbachia-free mosquitos will die. This counterintuitive behaviour may be explained by the relatively big size of $n_{2}$ with respecto to $n_{1}$ which makes population $n_{2}$ to have weak effect on the logistic grow of $n_{1}$.

Example 1. Take for instance $b_{1}=0.8, b_{2}=0.6, d_{1}=0.27, d_{2}=0.3, s_{h}=$ 0.8 , and $K=1$ as in [1]. Take $g_{2}=0.09(2+\sin (2 \pi t))$. Here $g_{2 *}=0.09$. Condition (20) does hold. The conclusion of Theorem 4.2 can be adopted. In Figure 1 we show numerical evidence with Mathematica supporting the existence of a stable limit cycle. We also have, $n_{1} \rightarrow 0$ for every initial condition. 


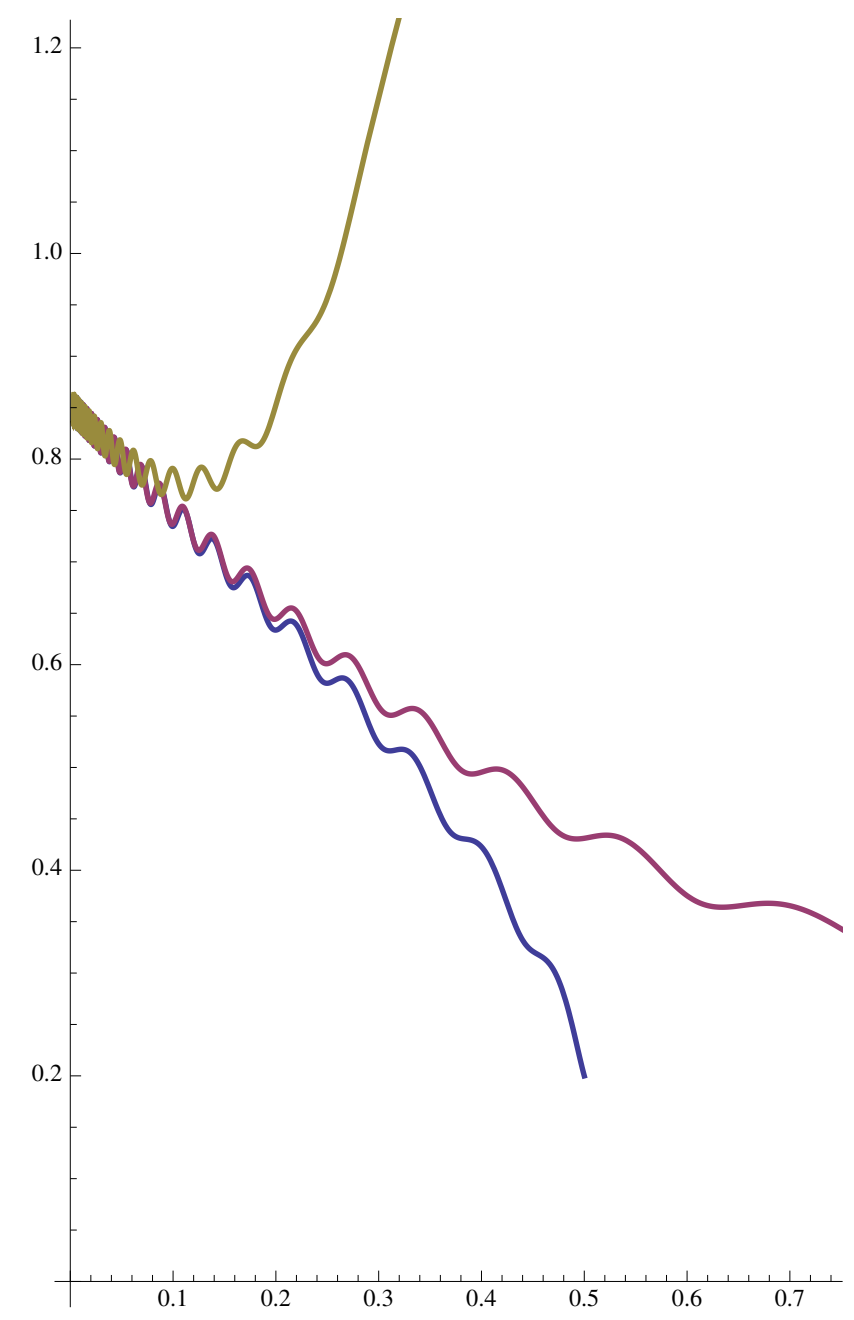

Figure 1: Plot of the solutions in phase space $\left(n_{1}, n_{2}\right)$ for several initial conditions $\left(n_{1}(0), n_{2}(0)\right) \in\{(0.5,0.2),(10,1),(1,10)\}$.

Example 2. Modify example 1 by $d_{2}=30$ and $g_{2}=0.9(2+\sin (2 \pi t))$. Here condition (20) does not hold. Numerical evidence suggest that a there may exist several distinct limit cycles, see Figure 2. In particular there may exist initial conditions for which Wolbachia-free population does not vanish. Here $b_{0} \approx 0.05898<K$ and condition 23 is not true. 


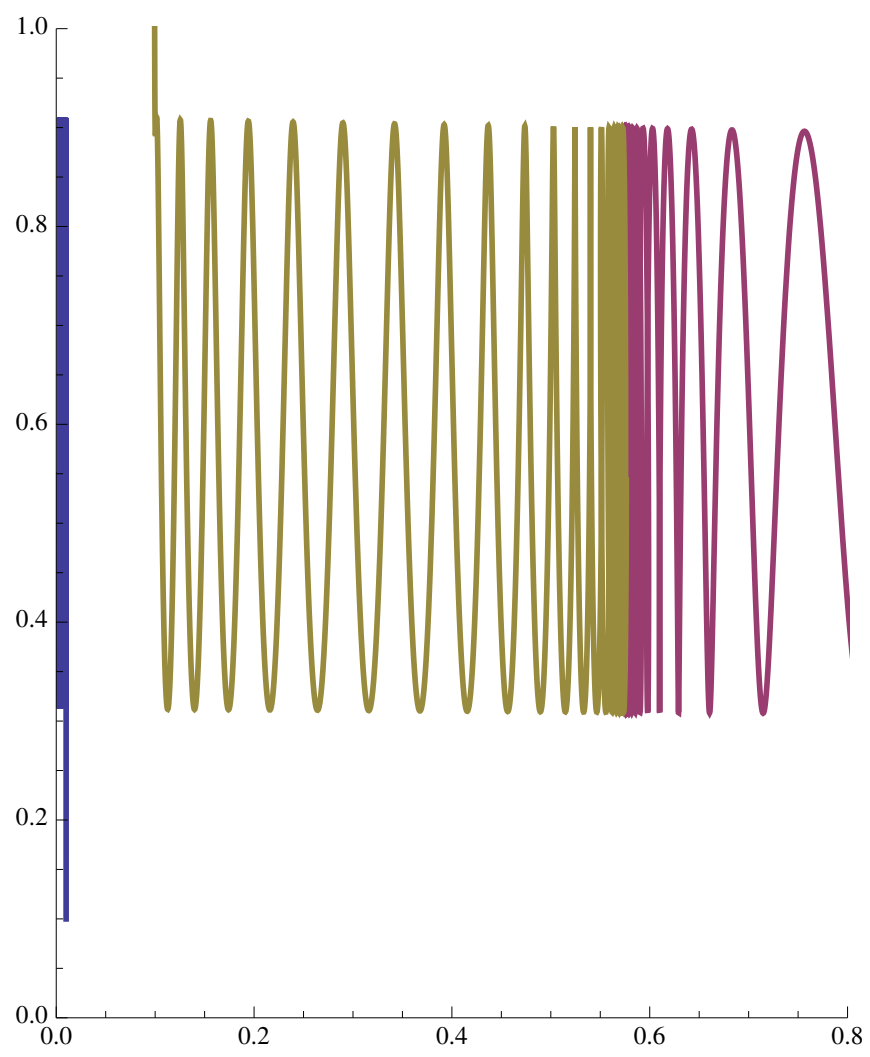

Figure 2: Plot of the solutions in phase space $\left(n_{1}, n_{2}\right)$ for several initial conditions $\left(n_{1}(0), n_{2}(0)\right) \in\{(0.01,0.01),(1,1),(0.1,1)\}$. There seems to be at least two limit cycles (there is a scale factor $10^{-1}$ in $n_{2}$ ).

Example 3. Modify example 1 by $d_{2}=0.03$. In this case (20) does hold, but Theorem 4.2 can not be applied. Nevertheless, numerical evidence suggest that there exists a global attractor which is limit cycle for which $n_{1} \rightarrow 0$, see Figure 3 . Here $b_{0} \approx 64.9>K$.

\section{Outlook}

Along this work we have extended the results of [6] from cooperative to competitive systems. Some existence results may be obtained in the case of competitive systems. There remains the question of estimating the number of periodic cycles existing when there is no unicity. Implications of different limit cycles for non vanishing Wolbachia-free $n_{1}$ population can also be of biological interest. 


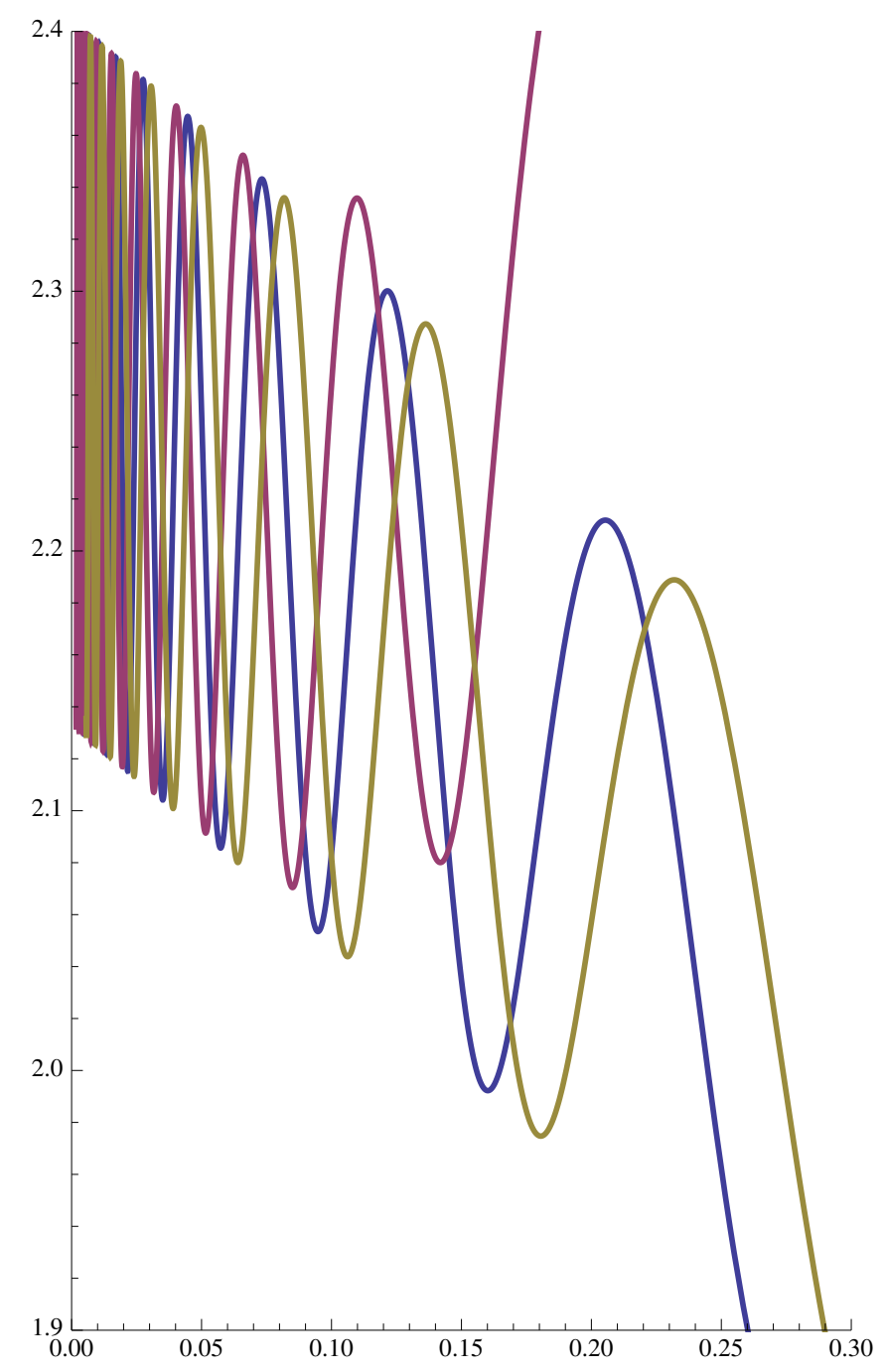

Figure 3: Plot of the solutions in phase space $\left(n_{1}, n_{2}\right)$ for several initial conditions $\left(n_{1}(0), n_{2}(0)\right) \in\{(0.8,0.1),(0.25,2.5),(1,0.1)\}$. There seems to be a global attracting limit cycle.

\section{Acknowledgements}

We thank the anonymous referees for some important suggestions.

Rev.Mate.Teor.Aplic. (ISSN print: 1409-2433; online: 2215-3373) Vol. 29(1): 53-68, Jan-Jun 2022 
H. G. DÍAZ-MARÍN - O. OSUNA

\section{Funding}

This work was partially supported by CONACYT-México and by Coordinación de la Investigación Científica Universidad Michoacana.

\section{References}

[1] L. Almeida, Y. Privat, M. Strugarek, N. Vauchelet, Optimal releases for population replacement strategies: Application to Wolbachia, SIAM Journal on Mathematical Analysis 51(2019), no. 4, 3170-3194. Doi: $10.1137 / 18 \mathrm{M} 1189841$

[2] H. Díaz-Marín, C.O. Osuna Castro, Periodic solutions for a model of cell population subjected to general periodic radiation, Revista Integración 38(2020), no. 2, 81-91. Doi: 10.18273/revint.v38n2-20200001

[3] A. d'Onofrio, A. Gandolfi, Tumour eradication by antiangiogenic therapy: analysis and extensions of the model by Hahnfeldt et al. (1999), Mathematical Biosciences 191(2004), no. 2, 159-184. Doi: $10.1016 /$ j.mbs.2004.06.003

[4] P. Hahnfeldt, D. Panigrahy, J. Folkman, L. Hlatky, Tumor development under angiogenic signaling: a dynamical theory of tumor growth, treatment response, and postvascular dormancy, Cancer research 59(1999), no. 19, 4770-4775.

[5] M.W. Hirsch, Systems of differential equations that are competitive or cooperative. V. Convergence in 3-dimensional systems, Journal of differential equations 80(1989), no. 1, 94-106. Doi: 10.1016/0022-0396(89)90097-1

[6] P. Korman, A periodic model for the dynamics of cell volume, arXiv, 2016. In: 1605.01324, 2016 [math.DS]

[7] H.L. Smith, Dynamics of competition, in: V. Capasso (Eds.) Mathematics Inspired by Biology, Lecture Notes in Mathematics 1714, Springer, Berlin, 1999, pp. 191-240. Doi: 10.1007/BFb0092378 